\title{
Hybrid Percutaneous-Endoscopic Removal (HPER) of Cholelithiasis
}

\author{
C. Roberto Simons-Linares ${ }^{a}$ Ram Gurajala $^{b}$ Gareth Morris-Stiff ${ }^{c}$ \\ Prabhleen Chahal $^{\mathrm{a}}$
}

${ }^{a}$ Gastroenterology and Hepatology Department, Digestive Disease Institute, Cleveland Clinic Foundation, Cleveland, $\mathrm{OH}, \mathrm{USA}^{\mathrm{b}}{ }^{\mathrm{b}}$ Interventional Radiology Department, Imaging Institute, Cleveland Clinic Foundation, Cleveland, $\mathrm{OH}, \mathrm{USA} ;{ }^{C}$ Hepatobiliary Surgery Department, Digestive Disease Institute, Cleveland Clinic Foundation, Cleveland, $\mathrm{OH}$, USA

\section{Keywords}

Acute cholecystitis - Endoscopic removal of gallstones -

Cholelithiasis - Gallbladder drainage · Recurrence of

cholecystitis · High-risk surgical candidate

\section{Abstract}

Acute cholecystitis (AC) affects over 20 million Americans annually, leading to annual costs exceeding USD 6 billion. Optimal treatment is early cholecystectomy. However, patients deemed high surgical risk undergo percutaneous cholecystostomy tube (PCT) placement as a bridge to surgery or more commonly as a definitive therapy. We hereby describe our experience with a new procedure named "Hybrid Percutaneous Endoscopic Removal (HPER) of cholelithiasis" that is meant for patients with chronic indwelling PCT. This procedure is an effective alternative to EUS-guided gallbladder drainage in high-risk patients. It does not require special expertise or technology and is simply performed by placing a fully covered metal stent conduit through the existing mature percutaneous tract allowing the endoscopic removal of gallstones through this conduit. This procedure can prevent the recurrence of gallstone-related complications as well as chronic PCT-related costs and adverse events. In our video, we present a case series and long-term follow-up of patients who underwent HPER as an alternative definitive therapy for calculous AC.

(c) 2020 S. Karger AG, Base

\section{Introduction}

Acute cholecystitis (AC) is one of the most common acute surgical conditions requiring hospitalization. AC affects over 20 million Americans annually leading to annual costs exceeding USD 6 billion [1]. Optimal treatment is early cholecystectomy (CCY). Patients deemed high-risk surgical candidates for CCY, undergo percutaneous cholecystostomy tube (PCT) placement as a bridge to surgery or as a definitive therapy. It has been reported that $50-70 \%$ of all patients continue to carry an indwelling PCT 1 year after initial placement $[2,3]$. Long-term use of indwelling catheters (i.e., PCTs) can be associated with significant morbidity, and it decreases the patient's quality of life $[4,5]$. We hereby report our experience with a simple and yet effective hybrid technique of percutaneous-endoscopic removal of cholelithiasis (HPER) [6]. The HPER procedure is an effective alternative to EUS-

karger@karger.com

www.karger.com/ddi

(C) 2020 S. Karger AG, Base

Karger $\stackrel{2}{=}$
Prabhleen Chahal, MD, FACG, FASGE

Gastroenterology and Hepatology Department, Digestive Disease Institute Cleveland Clinic Foundation, 9500 Euclid Avenue

Cleveland, OH 44195 (USA)

chahalp@ccf.org 


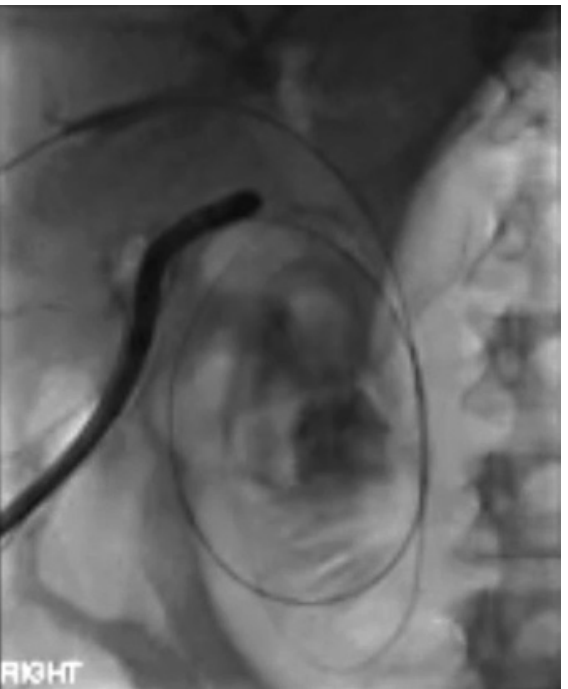

Video 1. Case series and long-term follow-up of patients who underwent hybrid percutaneous endoscopic removal of cholelithiasis as an alternative definitive therapy for calculous acute cholecystitis.

guided gallbladder drainage in high-risk patients. It does not require special expertise or technology and is simply performed by placing a fully covered metal stent conduit through the existing mature percutaneous tract allowing the endoscopic removal of gallstones through this conduit. HPER can prevent the recurrent of gallstone-related complications as well as PCT-related adverse events. Our case series reports this technique's effectiveness and longterm follow-up in patients who underwent HPER as an alternative definitive treatment for calculous AC.

\section{Video Description (Cases and Procedure)}

We present a case series of three critically ill patients who underwent PCT placement for calculous AC. After PCT placement, all three patients had recurrent biliary events (cholecystitis, cholangitis, biliary pancreatitis, biliary colic) and tube-related complications (migration, leak, bleeding, dislodgement, pain at the tube site) requiring re-interventions (video 1 ).

\section{Case 1}

A 76-year-old woman with severe cardiopulmonary comorbidities was referred to us because of persistent bile leakage around the PCT, requiring multiple PCT revisions, exchanges, and re-hospitalizations.

\section{Case 2}

A 47-year-old woman with borderline resectable pancreatic adenocarcinoma, who had acute perforated gangrenous cholecystitis with significant stone burden requiring PCT placement, was re- ferred to us. PCT placement was complicated by multiple indwelling catheter-related infections, cholangitis, and bile leaks. Importantly, the patient was deemed unfit for neoadjuvant chemotherapy and, hence, potential surgical resection due to recurrent biliary infections.

Case 3

A 64-year-old man was referred to us with complex cardiac (multiple myocardial infarctions, requiring multiple coronary interventions) and vascular (aortic, superior mesenteric artery repairs and stents) comorbidities who had calculous AC and had undergone PCT placement intraoperatively during an aborted laparoscopic-to-open converted cholecystectomy. His post-PCT course was complicated by recurrent biliary infections, requiring multiple PCT exchange, emergency room visits, and re-hospitalizations.

\section{Procedure Description}

Over a guidewire (Jagwire 0.035"; Boston Scientific, Marlborough, MA, USA) and under fluoroscopy guidance, the preexisting PCT tube is removed. An esophageal, fully covered self-expandable metal stent (SEMS) (diameter $16 \mathrm{~mm} \times$ length $8 \mathrm{~cm}$; Taewoong Medical, Gyeonggi-do, South Korea) (off-label use) is deployed by the advanced endoscopist over the guidewire across the preexisting mature percutaneous tract under fluoroscopy guidance with the proximal end in the gallbladder and the distal end protruding out from the cutaneous tract. The size of the stent was determined after reviewing available images (CT scan, MRI) and with the use of intra-procedural real-time fluoroscopy allowing the eventual measurement of the length of the catheter needed to reach the gallbladder from the percutaneous tract. Then, using a balloon dilator (Atlas; Bard Peripheral Vascular Inc., AZ, USA), the SEMS is dilated to $14-16 \mathrm{~mm}$. Following, the standard upper endoscope (GIF-HQ190; Olympus America Medical, PA, USA) is advanced through the SEMS into the gallbladder, and all gallstones are removed with the use of a roth net and/or basket (US Endoscopy, Mentor, OH, USA). After the complete removal of all gallstones, the SEMS is removed, standard dressing is applied at the exit site, and the procedure is concluded.

All three patients had calculous AC and were deemed unfit for surgery due to multiple comorbidities and therefore underwent PCT placement. The patients had recurrent symptoms of cholangitis and bile leaks, requiring multiple tube exchanges and hospitalizations, and all of them reported a significant decrease in quality of life. PCT placement was their long-term therapy due to persistence of severe comorbidities and severe stone burden; which precluded either removal of the PCT or CCY. The patients were looking for an alternative therapy and were referred to our clinic. We recommended the HPER procedure.

HPER was successfully performed in all three patients. Additionally, where indicated, common bile duct drainage with endoscopic retrograde cholangiopancreatography (ERCP) was simultaneously performed. All gallstones were successfully removed, and complete clearance of gallbladder, cystic duct, and common bile duct was achieved in all patients.

\section{Follow-Up}

The three patients continue to do well to date (longest followup: 4 years) without recurrence of biliary events and without any long-term HPER procedure-related complications. Of note, no 
cholecystocutaneous fistula (CCF), a rare complication after PCT occurring in $1-2 \%$ of all patients, was seen in any of our patients $[7,8]$. CCF formation may be related to ongoing inflammation from acute, recurrent, or chronic cholecystitis, or it can be formed due to long-standing, indwelling PCTs in the setting of atonic gallbladder, obstructed cystic duct, or common bile duct. In our series of reported cases, all gallstones were removed from the gallbladder, cystic duct and common bile duct were patent, and PCT was successfully discontinued without CCF formation.

\section{Implications and Conclusion}

The described procedure (HPER) is an attractive alternative for patients with chronic PCT who are unlikely to undergo CCY. A unique advantage of HPER over PCT and current endoscopic alternatives (i.e., transpapillary approach with ERCP) is the ability of complete gallstone(s) removal, hence preventing the recurrence of symptoms from persistent cholelithiasis $[9,10]$. EUSguided transmural gallbladder drainage also allows the removal of gallstones through a lumen-apposing metal stent, but it is a relatively new procedure, which requires expert-skills, and is not currently available across the majority of hospitals in the USA.

In summary, HPER is a safe, effective, and definitive treatment method for symptomatic cholelithiasis in highrisk surgical patients with chronic PCT. This case series demonstrated a long follow-up without recurrent gallbladder symptoms. It can improve morbidity and, most importantly, the patient's quality of life.

\section{Statement of Ethics}

This paper complies with the guidelines for human studies and was conducted ethically in accordance with the World Medical Association Declaration of Helsinki. Written informed consent was obtained.

\section{Disclosure Statement}

All authors have no conflicts of interest or financial ties to disclose.

\section{Funding Sources}

The authors have no funding sources to report.

\section{Author Contributions}

C. Roberto Simons-Linares: conceptualization, data collection, manuscript drafting and editing, video editing, supervision, final revision, and approval. Ram Gurajala: performed procedure, manuscript revision, video revision, and supervision. Gareth MorrisStiff: performed procedure, manuscript revision, video revision, and supervision. Prabhleen Chahal: conceptualization, performed procedure, manuscript revision, video revision, supervision, final revision, and approval.

\section{References}

1 Stinton LM, Shaffer EA. Epidemiology of gallbladder disease: cholelithiasis and cancer. Gut Liver. 2012 Apr;6(2):172-87.

2 Alvino DM, Fong ZV, McCarthy CJ, Velmahos G, Lillemoe KD, Mueller PR, et al. LongTerm Outcomes Following Percutaneous Cholecystostomy Tube Placement for Treatment of Acute Calculous Cholecystitis. J Gastrointest Surg. 2017 May;21(5):761-9.

3 Wang $\mathrm{CH}$, Wu CY, Yang JC, Lien WC, Wang HP, Liu KL, et al. Long-Term Outcomes of Patients with Acute Cholecystitis after Successful Percutaneous Cholecystostomy Treatment and the Risk Factors for Recurrence: A Decade Experience at a Single Center. PLoS One. 2016 Jan;11(1):e0148017.
4 Bundy J, Srinivasa RN, Gemmete JJ, Shields JJ, Chick JF. Percutaneous Cholecystostomy: Long-Term Outcomes in 324 Patients. Cardiovasc Intervent Radiol. 2018 Jun;41(6): 928-34.

5 McKay A, Abulfaraj M, Lipschitz J. Short- and long-term outcomes following percutaneous cholecystostomy for acute cholecystitis in high-risk patients. Surg Endosc. 2012 May; 26(5):1343-51.

6 Patel M, Levitin A, Chahal P. Hybrid percutaneous-endoscopic drainage of cholelithiasis and choledocholithiasis. Gastrointest Endosc. 2015 Apr;81(4):1013-4.

7 Horn T, Christensen SD, Kirkegård J, Larsen LP, Knudsen AR, Mortensen FV. Percutaneous cholecystostomy is an effective treatment option for acute calculous cholecystitis: a 10year experience. HPB (Oxford). 2015 Apr; 17(4):326-31.
8 Peters R, Kolderman S, Peters B, Simoens M, Braak S. Percutaneous cholecystostomy: single centre experience in 111 patients with an acute cholecystitis. JBR-BTR. 2014 Jul-Aug; 97(4):197-201.

9 Salameh H, DiMaio CJ. Endoscopic Retrograde Cholangiopancreatography and Endoscopic Ultrasound-Guided Gallbladder Drainage. Gastrointest Endosc Clin N Am. 2019 Apr;29(2):293-310.

10 Beland MD, Patel L, Ahn SH, Grand DJ. Image-Guided Cholecystostomy Tube Placement: Short- and Long-Term Outcomes of Transhepatic Versus Transperitoneal Placement. AJR Am J Roentgenol. 2019 Jan;212(1): 201-4. 